\title{
Inclusion of a Direct and Inverse Energy-Consistent Hysteresis Model in Dual Magnetostatic Finite Element Formulations
}

\author{
K. Jacques ${ }^{1,2}$, R. V. Sabariego ${ }^{3}$, C. Geuzaine ${ }^{2}$, and J. Gyselinck ${ }^{1}$ \\ ${ }^{1}$ BEAMS Department, Université Libre de Bruxelles (ULB), Belgium \\ ${ }^{2} \mathrm{ACE}$, Dept. of Electrical Engineering and Computer Science, University of Liège (ULg), Belgium \\ ${ }^{3}$ Dept. Electrical Engineering (ESAT), EnergyVille, KU Leuven, Belgium
}

\begin{abstract}
This paper deals with the implementation of an energy-consistent ferromagnetic hysteresis model in 2D finite element computations. This vector hysteresis model relies on a strong thermodynamic foundation and ensures the closure of minor hysteresis loops. The model accuracy can be increased by controlling the number of intrinsic cell components while parameters can be easily fitted on common material measurements. Here, the native $\boldsymbol{h}$-based material model is inverted using the Newton-Raphson method for its inclusion in the magnetic vector potential formulation. Simulations are performed on a 2D T-shaped magnetic circuit exhibiting rotational flux. By way of validation, results are compared with those obtained with the dual magnetic scalar potential formulation. A very good agreement for global quantities is observed.
\end{abstract}

Index Terms-Finite element analysis, Magnetic hysteresis, Newton Method.

\section{INTRODUCTION}

$\mathbf{I}$ $\mathrm{N}$ THE domain of numerical electromagnetism, increasing attention is paid to the modeling of ferromagnetic hysteretic materials with the aim of predicting the iron losses with high accuracy. However, the inclusion of a hysteresis model in a finite element (FE) computation remains challenging due to strong nonlinearities and potential inconsistencies between the input vector variable of the model (magnetic field $\boldsymbol{h}$ or induction field $\boldsymbol{b}$ ) and the basic variable of the FE formulation (e.g. magnetic scalar potential $\phi$, magnetic vector potential $\boldsymbol{a}$ ).

The aim of this work is to incorporate an energy-consistent hysteresis model into a 2D FE model with the classical onecomponent magnetic vector potential (MVP) formulation.

In the first section of this paper, the considered hysteresis model is briefly presented in its original direct form, driven by the magnetic field $\boldsymbol{h}$ as input. This $\boldsymbol{h}$-based model has already been presented in [1], [2], [3], works inspired notably by [4].

However, since the MVP-formulation has the induction field $\boldsymbol{b}$ as unknown field, the hysteresis model needs to be driven by the variable $\boldsymbol{b}$ instead. This inversion is done with the NewtonRaphson (NR) technique detailed in the second section.

Finally, FE simulations with the MVP-formulation (inverse hysteresis model included) are carried out on a T-joint of a three-phase transformer and compared with results obtained with the dual magnetic scalar potential (MSP) formulation (direct model included).

\section{Energy-Consistent Hysteresis Model}

The magnetic hysteresis finds its physical origin at the level of Weiss domains with the pinning effect of Bloch walls around defects in the material structure. The energy-consistent

Manuscript received July 6, 2015; revised August 20, 2015; accepted October 2, 2015. Date of publication Month Day, Year; date of current version October 12, 2015. Corresponding author: K. Jacques (email: Kevin.Jacques@doct.ulg.ac.be). Digital Object Identifier (inserted by IEEE). hysteresis model [1], [2] which has similarities with the one presented in [4], is based on the analogy between this pinning effect and the dry friction in mechanics so that it has a simple mechanical representation formed by the parallel connection of a spring and a friction slider. The statistical distribution of the pinning field that is specific to each material and characterizes most of its hysteretic behavior is discretized and incorporated into the model by considering several spring-friction slider cells connected in series (see Fig. 1). The applied force is analogous to the magnetic field $\boldsymbol{h}$ while the elongation is the magnetic polarization $\boldsymbol{J}$ that is split up in $N$ components spread over $N$ cells $\left(\boldsymbol{J}=\sum_{k} \boldsymbol{J}^{k}\right)$. The friction sliders, which model the pinning effect, are unlocked when the applied field exceeds a threshold specific to each cell and denoted $\chi^{k}$. On the other hand, the energy $u^{k}$ in the springs corresponds to the magnetic energy stored in the material. The springs thus account for the reversible part of the material response while the friction sliders account for the irreversible one. To some extent, this friction-like hysteresis model can also be seen as a vector version of series-connected play-hysteronbased type models [5]. However, conventional play-hysteron model types like Preisach or Prandtl-Ishilinskii have no real interpretation in terms of energy while the considered model relies consistently on an energy balance at each instant.

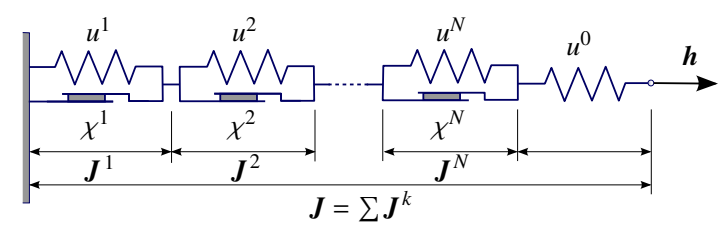

Fig. 1. Mechanical analogy of the hysteresis model with $\mathrm{N}$ cells.

There are two implementations of the energy-consistent hysteresis model, both controlled by the magnetic field $\boldsymbol{h}$ in their natural formulation. The first, differential [1], is rigorous 
in the 1D case, but comprises a small approximation when used in 2D or 3D cases. This simplification also appears in [4], where the direction of the magnetization change is somewhat fixed a priori. The second implementation [2], variational, avoids this approximation and consists in building for each cell a functional $\Omega^{k}$, the minimization of which at each instant amounts to finding the material polarization components $\boldsymbol{J}^{k}$. This functional relies on a consistent thermodynamic background and reads

$$
\Omega^{k}\left(\boldsymbol{h}, \boldsymbol{J}^{k}, \boldsymbol{J}_{p}^{k}\right)=u^{k}\left(\left|\boldsymbol{J}^{k}\right|\right)-\boldsymbol{h} \cdot \boldsymbol{J}^{k}+\chi^{k}\left|\boldsymbol{J}^{k}-\boldsymbol{J}_{p}^{k}\right| .
$$

where $\boldsymbol{J}_{p}^{k}$ represents the magnetic polarization field at the previous instant and contains the magnetic history of the material response. The pinning field $\chi^{k}$ is represented by a scalar value. The energy density $u^{k}$ is defined by the integration of a scalar saturation curve

$$
u^{k}\left(\left|\boldsymbol{J}^{k}\right|\right):=\int_{0}^{\left|J^{k}\right|} \alpha \operatorname{atanh}\left(\frac{J^{\prime}}{J_{s}^{k}}\right) \mathrm{d} J^{\prime},
$$

with $J_{s}^{k}$ the saturation magnetic polarization, and $\alpha$ a parameter inversely proportional to the slope of the curve at the origin. Here, the atanh function has been chosen to phenomenologically represent anhysteretic magnetization but any other function with appropriate shape could be used as well, e.g. the double Langevin function. The updated values $\boldsymbol{J}^{k}$ follow from minimizing separately each independent $\Omega^{k}(1)$ :

$$
\boldsymbol{J}^{k}=\mathcal{J}^{k}\left(\boldsymbol{h}, \boldsymbol{J}_{p}^{k}\right)=\underset{\boldsymbol{J}^{k}}{\operatorname{argmin}} \Omega^{k}\left(\boldsymbol{h}, \boldsymbol{J}^{k}, \boldsymbol{J}_{p}^{k}\right) .
$$

The magnetic induction field $\boldsymbol{b}$ can then be computed:

$$
\boldsymbol{b}=\mathcal{B}\left(\boldsymbol{h}, \boldsymbol{J}_{p}^{k}\right)=\mu_{0} \boldsymbol{h}+\sum \mathcal{J}^{k}\left(\boldsymbol{h}, \boldsymbol{J}_{p}^{k}\right),
$$

where $\mu_{0}$ is the magnetic permeability of vacuum.

Compared to other classical hysteresis models (Preisach and Jiles-Atherton (JA)) [6], [7], [8], the energy-consistent model is built without making assumptions about the applied field and is thus naturally a vector model. Based on an energy representation of the hysteresis phenomenon, the stored energy density and the dissipated power density (losses) are readily accessible at any instant. Moreover, the closure of minor hysteresis loops is guaranteed contrary to the JA model. Finally, the choice of the number of cells allows for a tradeoff between accuracy and complexity. Therefore, unlike the JA model, the number of parameters is not fixed a priori and can be straightforwardly identified from common material measurements (Epstein frame) [3].

As illustration, a 3-cell hysteresis model fitted on the basis of Epstein measurements carried out on the electrical steel M250-50A [2] is adopted; $b h$-curves obtained with multiharmonic and rotational $\boldsymbol{h}$ excitations are shown in Fig. 2. A 3-cell model gives satisfactory results for simple excitations whereas in presence of higher harmonics, 7 cells may be needed.

\section{Model InVersion with Newton-Raphson}

In order to include the energy-consistent hysteresis model in $\mathrm{FE}$ formulations with the magnetic induction field $\boldsymbol{b}$ as

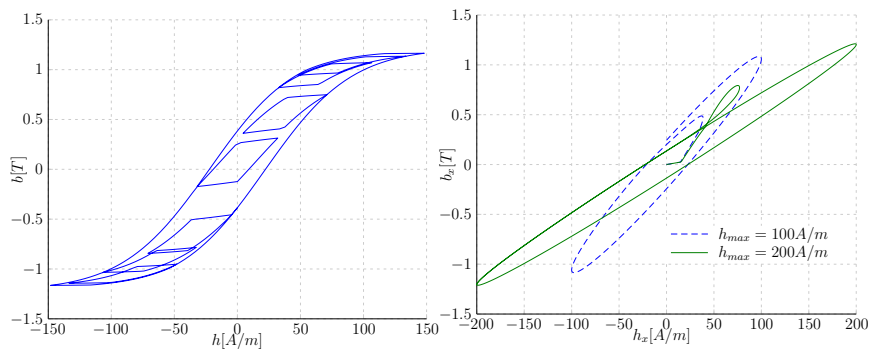

Fig. 2. Multi-harmonic (left) and rotational excitations (right) with 3 cells: $\left[J_{s}^{1}=0.11 T ; \chi^{1}=0 A / m\right]\left[J_{s}^{2}=0.8 T ; \chi^{2}=16 A / m\right]\left[J_{s}^{3}=0.31 T ; \chi^{3}=47 A / m\right]$.

unknown (e.g. MVP-formulation), it must be driven with the variable $\boldsymbol{b}$ instead of $\boldsymbol{h}$. Moreover, the possibility of handling both $\boldsymbol{b}=\mathcal{B}(\boldsymbol{h})$ and $\boldsymbol{h}=\mathcal{H}(\boldsymbol{b})$ relations enables to tackle complementary formulations for the numerical solution of magnetic field problems [9].

For a given input vector $\boldsymbol{b}^{*}$, the Newton-Raphson (NR) technique consists in finding increasingly better approximations of the field $\boldsymbol{h}^{*}$ that verifies $\boldsymbol{r}\left(\boldsymbol{h}^{*}\right)=\mathcal{B}\left(\boldsymbol{h}^{*}, \boldsymbol{J}_{p}^{k}\right)-\boldsymbol{b}^{*}=\mathbf{0}$, with $\mathcal{B}$ given by the direct model (4). Starting from an initial estimate $\boldsymbol{h}_{0}$, the NR process produces subsequent increments $\Delta \boldsymbol{h}_{i+1}$, and so the next estimated magnetic field values $\boldsymbol{h}_{i+1}=\boldsymbol{h}_{i}+\theta \Delta \boldsymbol{h}_{i+1}$, with a relaxation factor $\theta \in] 0,1$ ], linearising $\boldsymbol{r}\left(\boldsymbol{h}_{i+1}\right)$ around the current known approximation $\boldsymbol{h}_{i}$ :

$$
\Delta \boldsymbol{h}_{i+1}=\left[\frac{\partial \mathcal{B}}{\partial \boldsymbol{h}}\left(\boldsymbol{h}_{i}, \boldsymbol{J}_{p}^{k}\right)\right]^{-1} \cdot\left(\boldsymbol{b}^{*}-\mathcal{B}\left(\boldsymbol{h}_{i}, \boldsymbol{J}_{p}^{k}\right)\right),
$$

until sufficient convergence, i.e. $\left|\boldsymbol{r}\left(\boldsymbol{h}_{i}\right)\right|<\epsilon$.

The differential permeability tensor $\frac{\partial \mathcal{B}}{\partial \boldsymbol{h}}$ is obtained by differentiating (4) with respect to $\boldsymbol{h}$ :

$$
\frac{\partial \mathcal{B}}{\partial \boldsymbol{h}}\left(\boldsymbol{h}, \boldsymbol{J}_{p}^{k}\right)=\mu_{0} \mathbb{I}+\sum \frac{\partial \mathcal{J}^{k}}{\partial \boldsymbol{h}}\left(\boldsymbol{h}, \boldsymbol{J}_{p}^{k}\right) .
$$

The analytical expression of the relative susceptibility tensors $\frac{\partial \mathcal{J}^{k}}{\partial h}$ can be deduced by exploiting the fact that in order to ensure (3), gradients of the functionals $\Omega^{k}(1)$ should be equal to zero if they exist. Particularly if the energy density function is modeled by (2), they read:

$$
\frac{\partial \Omega^{k}}{\partial \boldsymbol{J}^{k}}\left(\boldsymbol{h}, \boldsymbol{J}^{k}, \boldsymbol{J}_{p}^{k}\right)=\alpha \operatorname{atanh}\left(\frac{\left|\boldsymbol{J}^{k}\right|}{\boldsymbol{J}_{s}^{k}}\right) \frac{\boldsymbol{J}^{k}}{\left|\boldsymbol{J}^{k}\right|}-\boldsymbol{h}+\chi^{k} \frac{\boldsymbol{J}^{k}-\boldsymbol{J}_{p}^{k}}{\left|\boldsymbol{J}^{k}-\boldsymbol{J}_{p}^{k}\right|}=\mathbf{0}
$$

As one can see from (7), the gradients are not analytically defined at angulous points $\left(\left|\boldsymbol{J}^{k}\right|=0\right.$ or $\left.\left|\boldsymbol{J}^{k}-\boldsymbol{J}_{p}^{k}\right|=0\right)$. Except for these specific points, relation (7), from (3), holds. Once again, by differentiation of (7) with respect to $\boldsymbol{h}$, an analytical form of the tensors $\frac{\partial \boldsymbol{J}^{k}}{\partial \boldsymbol{h}}=\frac{\partial \mathcal{J}^{k}}{\partial \boldsymbol{h}}\left(\boldsymbol{h}, \boldsymbol{J}_{p}^{k}\right)$ can finally be extracted:

$$
\begin{array}{r}
\frac{\partial \boldsymbol{J}^{k}}{\partial \boldsymbol{h}}=\left(\frac{\alpha J_{s}^{k}}{\left(J_{s}^{k}\right)^{2}-\left|\boldsymbol{J}^{k}\right|^{2}} \frac{\boldsymbol{J}^{k} \boldsymbol{J}^{k^{T}}}{\left|\boldsymbol{J}^{k}\right|^{2}}+\frac{\alpha}{\left|\boldsymbol{J}^{k}\right|} \operatorname{atanh}\left(\frac{\left|\boldsymbol{J}^{k}\right|}{\boldsymbol{J}_{s}^{k}}\right)\left(\mathbb{I}-\frac{\boldsymbol{J}^{k} \boldsymbol{J}^{T}}{\left|\boldsymbol{J}^{k}\right|^{2}}\right)\right. \\
\left.+\frac{\chi^{k}}{\left|\boldsymbol{J}^{k}-\boldsymbol{J}_{p}^{k}\right|}\left(\mathbb{I}-\frac{\left(\boldsymbol{J}^{k}-\boldsymbol{J}_{p}^{k}\right)\left(\boldsymbol{J}^{k}-\boldsymbol{J}_{p}^{k}\right)^{T}}{\left|\boldsymbol{J}^{k}-\boldsymbol{J}_{p}^{k}\right|^{2}}\right)\right)^{-1},
\end{array}
$$

where $\boldsymbol{J}^{k}$ depends on $\boldsymbol{h}$ and $\boldsymbol{J}_{p}^{k}$ as computed from (3). Introducing (8) into (6) allows to analytically calculate the Jacobian matrix $\frac{\partial \mathcal{B}}{\partial h}$. At angulous points, (8) does not hold anymore and is therefore numerically approximated by finite 
differences. The same difficulties are encountered in [10] where the differentiation of a vector play hysteretic function is discussed and a practical way to tackle the problem is given.

Nevertheless, despite the presence of discontinuities in the differentiation, the NR inversion at the material level works quite well without relaxation factor. Some convergence problems may be encountered near magnetic saturation, when $\left|\boldsymbol{J}^{k}\right|$ values are close to their maximum $J_{s}^{k}$ so that the relative susceptibility tensors (8) tend to become singular. For the studied material (Fig. 2), this may happen when $|\boldsymbol{h}|>470 \mathrm{~A} / \mathrm{m}$, as for higher fields: $\left(\left|\boldsymbol{J}^{k}\right|-J_{s}^{k} \mid\right)<1 e-6 \cdot J_{s}^{k}$. Using a relaxation factor $(\theta<1)$ or increasing the discretization in time may help the NR process to converge in that case.

Finally, the inverse model can be summarized as follows:

$$
\boldsymbol{h}=\mathcal{H}\left(\boldsymbol{b}, \boldsymbol{J}_{p}^{k}\right)=\underset{\boldsymbol{h}}{\arg \min }\left|\mathcal{B}\left(\boldsymbol{h}, \boldsymbol{J}_{p}^{k}\right)-\boldsymbol{b}\right|
$$

\section{Inclusion in Dual 2D FE Formulations}

\section{A. MVP-Formulation}

As in [11] for the Jiles-Atherton model, the inverse model (9) is included in the 2D FE modeling with the magnetostatic $\boldsymbol{a}$-formulation, weak form of the Ampere law $\left(\operatorname{curl} \boldsymbol{h}=\boldsymbol{j}_{s}\right.$ ): find the MVP $\boldsymbol{a}=a_{z} \boldsymbol{e}_{z}$ such that

$$
\left(\mathcal{H}\left(\operatorname{curl} \boldsymbol{a}, \boldsymbol{J}_{p}^{k}\right), \operatorname{curl} \boldsymbol{a}^{\prime}\right)_{\Omega}+\left\langle\boldsymbol{h} \times \boldsymbol{n}, \boldsymbol{a}^{\prime}\right\rangle_{\Gamma}=\mathbf{0}
$$

holds for suitable test functions $\boldsymbol{a}^{\prime}$, where $(\cdot, \cdot)_{\Omega}$ and $\langle\cdot, \cdot\rangle_{\Gamma}$ denote the integral of the scalar product of the two arguments over a volume $\Omega$ and on its boundary $\Gamma$, respectively, and where $\boldsymbol{n}$ is the inward unit normal on $\Gamma$. No current density $\boldsymbol{j}_{s}$ is considered here $\left(\boldsymbol{j}_{s} \equiv \mathbf{0}\right)$. In practice, $\boldsymbol{a}$ is discretized with appropriate basis functions that are also used as test functions $\boldsymbol{a}^{\prime}$, applying the Galerkin method; the induction $\boldsymbol{b}=\operatorname{curl} \boldsymbol{a}$ satisfies exactly the flux conservation law $(\operatorname{div} \boldsymbol{b}=0)$.

Because of the hysteretic material behavior, after discretization, equation (10) must be time-stepped. Starting from a known solution $\boldsymbol{a}_{p}$ and known material state $\left(\boldsymbol{b}_{p}, \boldsymbol{h}_{p}, \boldsymbol{J}_{p}^{k}\right)$ from the previous instant, the solution at the current instant can be obtained by means of an iterative NR scheme at the FE level. At each nonlinear iteration, (10) is linearised and solved with respect to the increment $\Delta \boldsymbol{a}_{i+1}$ :

$$
\begin{gathered}
\left(\frac{\partial \mathcal{H}}{\partial \boldsymbol{b}}\left(\boldsymbol{h}_{i}, \boldsymbol{J}_{p}^{k}\right) \cdot \operatorname{curl} \Delta \boldsymbol{a}_{i+1}, \operatorname{curl} \boldsymbol{a}^{\prime}\right)_{\Omega}= \\
-\left\langle\boldsymbol{h} \times \boldsymbol{n}, \boldsymbol{a}^{\prime}\right\rangle_{\Gamma}-\left(\boldsymbol{h}_{i}, \operatorname{curl} \boldsymbol{a}^{\prime}\right)_{\Omega} .
\end{gathered}
$$

A new approximation is produced $\boldsymbol{a}_{i+1}=\boldsymbol{a}_{i}+\theta \Delta \boldsymbol{a}_{i+1}$ with relaxation factor $\theta \in] 0,1]$ to ensure convergence. This operation is repeated until a sufficiently satisfactory solution for (10) is achieved for the current time step.

The differential reluctivity tensor $\frac{\partial \mathcal{H}}{\partial b}$, inverse of the tensor $\frac{\partial \mathcal{B}}{\partial \boldsymbol{h}}$ shown in (6), emerges in (11). The notation $\boldsymbol{h}_{i}=$ $\mathcal{H}\left(\operatorname{curl} \boldsymbol{a}_{i}, \boldsymbol{J}_{p}^{k}\right)$ is used to represent the magnetic field updated by the inverse model (9) based on known values from the $i$-th iteration in the NR process at the FE level. Eventually, after convergence, magnetic polarization components $\boldsymbol{J}^{k}$ are updated thanks to (3). The new material state is thus completely defined and the next time step can be handled.

\section{B. MSP-Formulation}

Analogously, the direct model (4) is included in the dual 2D magnetostatic $\phi$-formulation, weak form of the flux conservation law $(\operatorname{div} b=0)$ : find the MSP $\phi$ such that

$$
\left(\mathcal{B}\left(-\operatorname{grad} \phi, \boldsymbol{J}_{p}^{k}\right), \operatorname{grad} \phi^{\prime}\right)_{\Omega}+\left\langle\boldsymbol{b} \cdot \boldsymbol{n}, \phi^{\prime}\right\rangle_{\Gamma}=0,
$$

holds for suitable test functions $\phi^{\prime}$. Again, the Galerkin method is applied; the magnetic field $\boldsymbol{h}=-\operatorname{grad} \phi$ satisfies exactly the Ampere law ( $\left.\operatorname{curl} \boldsymbol{h}=\boldsymbol{j}_{s} \equiv \mathbf{0}\right)$.

As before, the nonlinear system (12) is solved at each time step with an iterative NR scheme at the FE level:

$$
\left(\frac{\partial \mathcal{B}}{\partial \boldsymbol{h}} \cdot \operatorname{grad} \Delta \phi_{i+1}, \operatorname{grad} \phi^{\prime}\right)_{\Omega}=\left\langle\boldsymbol{b} \cdot \boldsymbol{n}, \phi^{\prime}\right\rangle_{\Gamma}+\left(\boldsymbol{b}_{i}, \operatorname{grad} \phi^{\prime}\right)_{\Omega},
$$

where the differential permeability tensor $\frac{\partial \mathcal{B}}{\partial \boldsymbol{h}}$ appears and $\boldsymbol{b}_{i}=$ $\mathcal{B}\left(-\operatorname{grad} \phi_{i}, \boldsymbol{J}_{p}^{k}\right)$ stands for the last known computed induction field value, requiring the use of the direct model (4).

\section{Boundary Conditions with Flux Walls and Flux Gates}

The boundary $\Gamma$ of the studied domain $\Omega$ is considered as an alternating series of flux walls $\Gamma_{w j}$ and flux gates $\Gamma_{g j}$ [12]. By definition, flux walls $\Gamma_{w j}$ are magnetically impermeable interfaces, on which $\boldsymbol{b} \cdot \boldsymbol{n}=0$ holds. The related magnetomotive forces are expressed by $\mathcal{F}_{j}=\left\langle\boldsymbol{h} \times \boldsymbol{n}, 1 \mathbf{e}_{\mathbf{z}}\right\rangle_{\Gamma_{w j}}$. On the other hand, flux gates $\Gamma_{g j}$ correspond to perfectly permeable interfaces, on which $\boldsymbol{h} \times \boldsymbol{n}=\mathbf{0}$ is verified. The magnetic fluxes flowing through the gates, inward $\Omega$, are given by $\Phi_{j}=\langle\boldsymbol{b} \cdot \boldsymbol{n}, 1\rangle_{\Gamma_{g j}}$. Therefore, the sum of the magnetomotive forces $\mathcal{F}_{j}$ is zero, as is the sum of the fluxes $\Phi_{j}$. An example with three flux walls and three flux gates from [12] is shown in Fig. 3

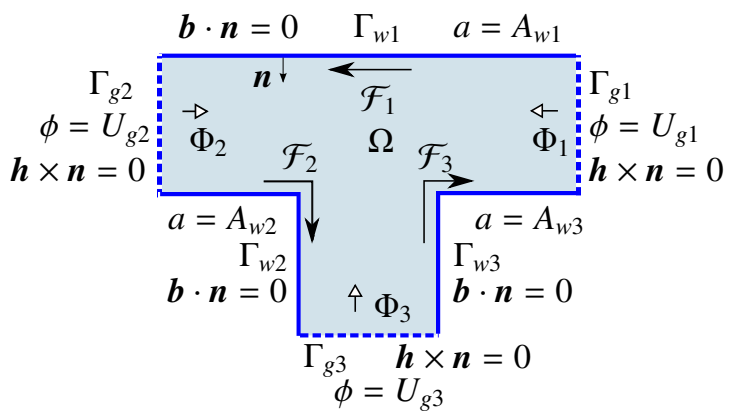

Fig. 3. Example of a sequence of three flux walls and three flux gates as boundary of a 2D model of a T-joint of a three-phase transformer [12].

In the MVP-formulation, $a$ has a constant value $A_{w j}$ along each flux wall $\Gamma_{w j}$. Gate fluxes between two walls can be strongly imposed by fixing the two associated $A_{w j}$ values as $\Phi_{j}=A_{w j}-A_{w k}$. Otherwise, an $A_{w j}$ value can be considered as a floating potential unknown of the problem. In that case, the linked magnetomotive force $\mathcal{F}_{j}$ is weakly imposed through the contour integral in (10). To this end, a specific basis function is used which decreases element-wise linearly from 1 on $\Gamma_{w j}$ to 0 in the layer of elements around $\Gamma_{w j}$.

Equivalently, in the MSP-formulation, $\phi$ can either be fixed a priori or constitutes a floating potential $\phi=U_{g j}$ on the corresponding flux gates $\Gamma_{g j}$. Therefore, in the latter case, the associated magnetic flux $\Phi_{j}$ is weakly imposed via the contour integral in (12). 


\section{Application example}

The 3-cell material hysteresis model presented in Fig. 2 is considered [2]. The MVP-formulation (11) as well as the MSP-formulation (13) are applied to the 2D FE model of the T-joint studied in Fig. 3. In order to validate the inverse hysteresis model developed in this paper, results from the MSP- and MVP-formulations, using the direct one (4) and the new inverse model (9) respectively, are compared.

A rotational field is effected by imposing strongly in the MVP- and weakly in the MSP-formulation the gate fluxes $\Phi_{1}=\cos (2 \pi f t+2 \pi / 3)$ and $\Phi_{2}=\cos 2 \pi f t$, with arbitrary frequency $f=1 \mathrm{~Hz}$. Two periods are sampled with 1000 time steps each. In order to start smoothly through the first magnetization curve of the hysteretic material, the fluxes $\Phi_{1}$ and $\Phi_{2}$ are multiplied during the first quarter of a period by the function $\left(1-\cos \left(\pi t / t_{\text {relax }}\right)\right) / 2$, with $t_{\text {relax }}=0.25 s$. The FE mesh is made of 729 triangles and yields 346 and 385 unknowns for MVP- $a e_{z}$ and MSP- $\phi$ respectively after the application of constraints.

The chosen stopping criteria for the NR processes at the FE level are $\left|\Delta x_{i}\right|_{1} /\left|x_{i}\right|_{1}<1 e-4$, with $x=a_{z}$ for MVP and $x=\phi$ for MSP $\left(\left.\left.\right|_{1}\right|_{1}\right.$ is the $L^{1}$-norm over all element unknowns). At each NR iteration, an optimal relaxation factor $\theta_{i}$ is selected between 10 evenly spaced values between 1 and 0.1 . Calculations are done in the open source environment Gmsh/GetDP. On average, the MVP problem converges with 3.83 iterations per time step ( 7 iterations for the worst step) while only 1 iteration is enough for the MSP problem at every time step.

The magnetomotive forces $\mathcal{F}_{1}(t)$ and $\mathcal{F}_{2}(t)$ associated to flux walls $\Gamma_{w 1}$ and $\Gamma_{w 2}$ respectively are shown in Fig. 4 (right). A very good agreement is observed between the results of both formulations. The $b$-loci and $h$-loci for the six points from Fig. 4 (left) are given in Fig. 5. Moreover, the $b_{x} h_{x}$ and $b_{y} h_{y}$ loops for these points are presented in Fig. 6.
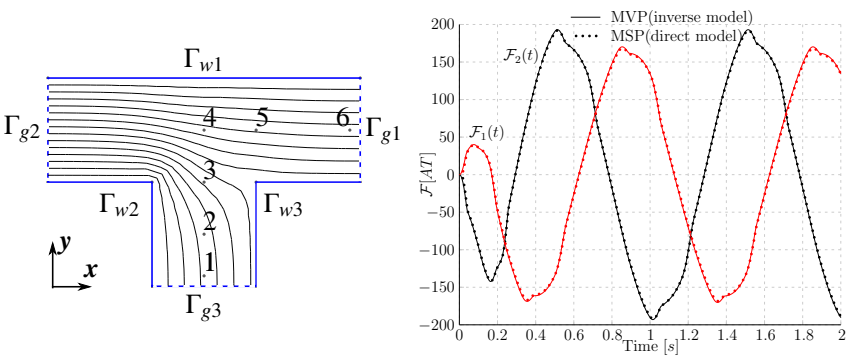

Fig. 4. (left): T-joint transformer with flux lines and location of six points (right): Magnetomotive forces $\mathcal{F}_{1}(t)$ (in red) and $\mathcal{F}_{2}(t)$ (in black)

\section{Conclusion}

The inclusion of an energy-consistent hysteresis model in 2D FE magnetic field problems with dual formulations has been realized. First, at the material level, the NR method has been used successfully to invert the naturally $\boldsymbol{h}$-based hysteresis model into a $\boldsymbol{b}$-based one. Then, at the FE level, once again, the NR technique has been adopted to solve the nonlinear equations, leading to the emergence of discontinuous differential reluctivity and permeability tensors.

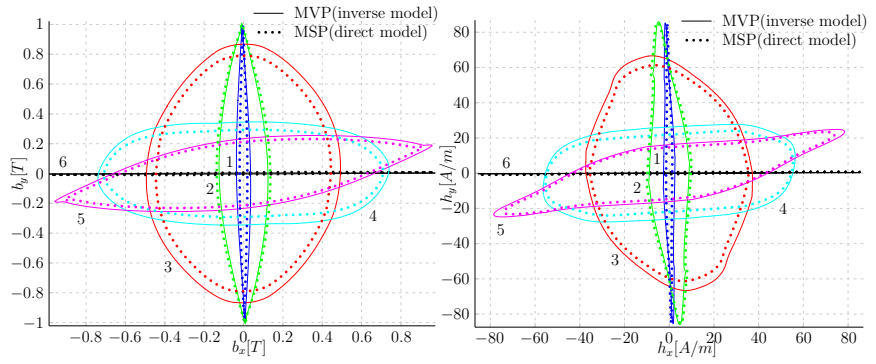

Fig. 5. $b$-loci (left) and $h$-loci (right) in the six points defined in Fig. 4 (left).
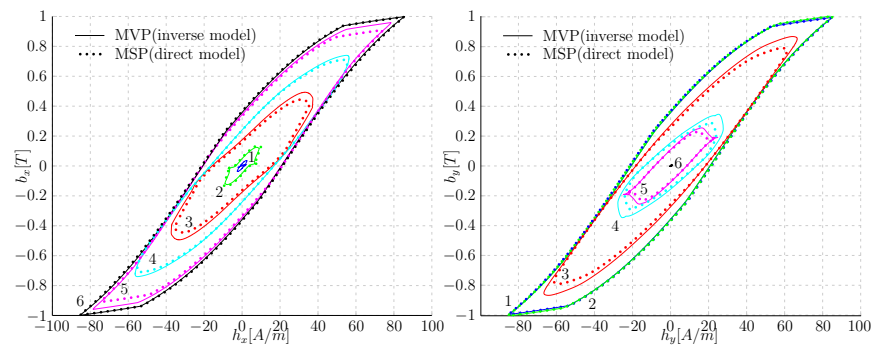

Fig. 6. $b_{x} h_{x}$ (left) and $b_{y} h_{y}$ (right) in the six points defined in Fig. 4 (left).

The two formulations have been applied to a simple 2D FE model with rotational excitation. Matching results between both complementary formulations have been observed.

\section{ACKNOWLEDGEMENTS}

This work was supported in part by the Belgian Science Policy under grant IAP P7/02 and the Walloon Region of Belgium under grant RW-1217703 (WBGreen FEDO).

\section{REFERENCES}

[1] F. Henrotte, A. Nicolet, K. Hameyer, "An energy-based vector hysteresis model for ferromagnetic model," Selected Papers from the EPNC 2004 Symp., Poznan, Poland, June 28-30, 2004.

[2] V. Franois-Lavet, F. Henrotte, L. Stainier, L. Noels, C. Geuzaine, "An energy-based variational model of ferromagnetic hysteresis for finite element computations," J. Comp. E Applied Math., vol. 246, pp. 243250,2013

[3] F. Henrotte, S. Steentjes, K. Hameyer, C. Geuzaine, "Iron Loss Calculation in Steel Laminations at High Frequencies," IEEE Trans. Mag., vol. 50, no. 2, pp. 333-336, 2014.

[4] A. Bergqvist, "Magnetic vector hysteresis model with dry friction-like pinning," Physica B, vol. 233, pp. 342-347, 1997.

[5] S. Bobbio, G. Miano, C. Serpico, and C. Visone, "Models of magnetic hysteresis based on play and stop hysterons," IEEE Trans. Magn., vol. 33, pp. 4417-4426, 1997.

[6] G. Bertotti, Hysteresis in Magnetism, Academic Press, 1998.

[7] I. Mayergoyz, Mathematical Models of Hysteresis and their Applications, Second Edition, Academic Press, 2003.

[8] D.C. Jiles, D.L. Atherton, "Theory of ferromagnetic hysteresis," Journal of Magnetism and Magnetic Materials, vol. 61, no. 1-2, pp. 48-60, 1986.

[9] J. Rikabi, C. F. Bryant, E. M. Freeman "An error-based approach to complementary formulations of static field solutions," Int. J. Numer. Methods Eng., vol. 26, p. 1963, 1988.

[10] R. Mitsuoka, T. Mifune, T. Matsuo, C. Kaido, "A vector play model for finite-element magnetic-field analysis with Newton-Raphson method," IEEE Trans. Magn., vol. 49, pp. 1689-1692, 2013.

[11] J. Gyselinck, P. Dular, N. Sadowski, J. Leite, J. P. A. Bastos, "Incorporation of a Jiles-Atherton vector hysteresis model in 2D FE magnetic field computations - Application of the NewtonRaphson method," COMPEL, vol. 23, no. 3, pp. 685-693, 2004.

[12] J. Gyselinck, L. Vandevelde , J. Melkebeek , P. Dular, "Complementary Two-dimensional Finite Element Formulations with Inclusion of a Vectorized Jiles-Atherton Model," COMPEL, vol. 23, no. 4, pp. 959967, 2004. 